\title{
Explicit discontinuum simulation for probabilistic forecasting of fault slip and rock mass seismic potential
}

\author{
D. Beck Beck Engineering Pty Ltd, Australia
}

V. Levkovitch Beck Engineering, Germany

B. Simser Xstrata Nickel Ltd., Canada

\section{Abstract}

With increasing depth, there will always be situations where stress, strength and structure combine to store energy in unstable ways around mining work areas. Some mining induced seismicity is unavoidable. The job of the planning team is to balance the compromises between production demands and rock mechanics driven sequencing principles so that all essential constraints-safety, economy and recovery-are met. Achieving a sufficient and defendable balance requires quantitative tools: tools that can differentiate various courses of action, as well as assess the underlying risks for each method. It is not good enough to simply rank possible scenarios or reduce the forecast performance to a single economic value, without actually determining if the bottom line risk is below acceptable levels.

In this paper, a numerical modelling technique for simulating seismic effects of mining is described. The approach estimates the energy released throughout the mine at each stope extraction step, and has been validated for its intended purpose using data from Xstrata's Nickel Rim South (NRS) Mine.

\section{Introduction}

The project aimed to develop a high resolution model to simulate the evolution of stress, strain and energy release between different structural and geotechnical domains in a complex mine geometry. The sufficiency requirement for the model was to match the measured seismicity well enough that the tool could be used to quantitatively differentiate potential levels of seismicity for alternative future mine sequences.

The method involved large strain, 3D discontinuum, strain softening dilatant modelling (Discontinuum Finite Element, or DFE modelling) of a detailed stope and development extraction sequence. The model contains all of the interpreted structure from the structural model and the interpreted geological domains without simplification. This detailed model was then run repeatedly, adjusting material properties and other constitutive variables in each run to eventually achieve the best possible match between modelled energy release and measured seismicity.

The end state of the project is a well calibrated model with well understood resolution and reliability and a future continuous improvement program for the model to facilitate integration with mine planning. 3D results have been provided to the mine to allow direct visualisation and comparison against the continuously generated field data, so that the model can be used for day-to-day geotechnical tasks, and also to allow continuous ground truthing.

The mine has implemented an ongoing program of testing, observation and model recalibration to improve the analysis and identify unforeseen seismic outcomes, and ensure that the most up to date seismic data is systematically considered when making planning decisions. 


\section{Model approach}

The key elements of the problem were the persistent and frequent faulting and the measurable rock mass degradation around drives, stopes and through secondary pillars.

The requirements of the problem constrained the analysis to 3D strain softening dilatant discontinuum simulation, which means that the rock mass in the model degrades due to over stressing, faults are represented as provided and can slip and separate, and the rock mass dilates as it degrades. The mining sequence also had to be represented in very small steps to replicate the stress path as closely as possible, including the sequential extraction of development to match the real schedule.

A model of this complexity consists of several parts:

- CAD: The models include all development, stopes and components of the geological and structural models without substantive simplification. The geometric complexity requires the use of modern CAD software to facilitate the sequencing and construction of the refined model geometry. Modelling software does not typically have the required functionality for this task.

- Meshing and model building using customised tools, which are necessary to incorporate the structural model without reduction in complexity because off the shelf modelling packages do not typically have the capacity to mesh complex excavation and geological geometries adequately or efficiently.

- Solution of the stress, strain and energy distribution using the Abaqus Explicit solver and the LR2 constitutive model (Levkovitch et al., 2010).

- Results visualisation using Voxler and the Abaqus Viewer.

\section{1 Constitutive model for the rock mass, faults and seismicity}

The LR2 model is a continuum/discontinuum constitutive model framework for discontinuum modelling. LR2's main features are:

1. The continuum parts (i.e. the rock mass between explicit structures) are modelled as a strain softening dilatant material. This means that as strain increases the material softens, weakens and dilates. Each geotechnical domain has its own set of material properties, and all parameters for each domain or fill can vary at different rates with respect to strain changes, including the dilatancy parameter. This allows approximation of complex stress-strain behaviour.

In a well calibrated strain softening model, rock mass damage is an output of the model, so can be directly compared to the observed damage in mine development.

2. All normal model outputs such as stress, displacement and strength loss are produced, but in addition, the plastic strain (damage) tensor is available. For ease of viewing, the dilation component of the plastic strain tensor is typically plotted as this is a scalar for which a simple colour scale can be used. It also correlates well with most operator's visual interpretation of damage.

For strong rock masses, the damage level is easily interpreted at the surface of excavations and settling on qualitative descriptions at a mine is usually simple. LR2s qualitative rock mass scale damage classification is shown in Figure 1.

The cubes of rock are at a $10 \mathrm{~m}$ scale, which is typical for hangingwall drive pillars at NRS Mine. The images show the typical equivalent structural damage and distortion in the rock mass, corresponding to levels of equivalent plastic strain in the continuum parts of an LR2 model. 


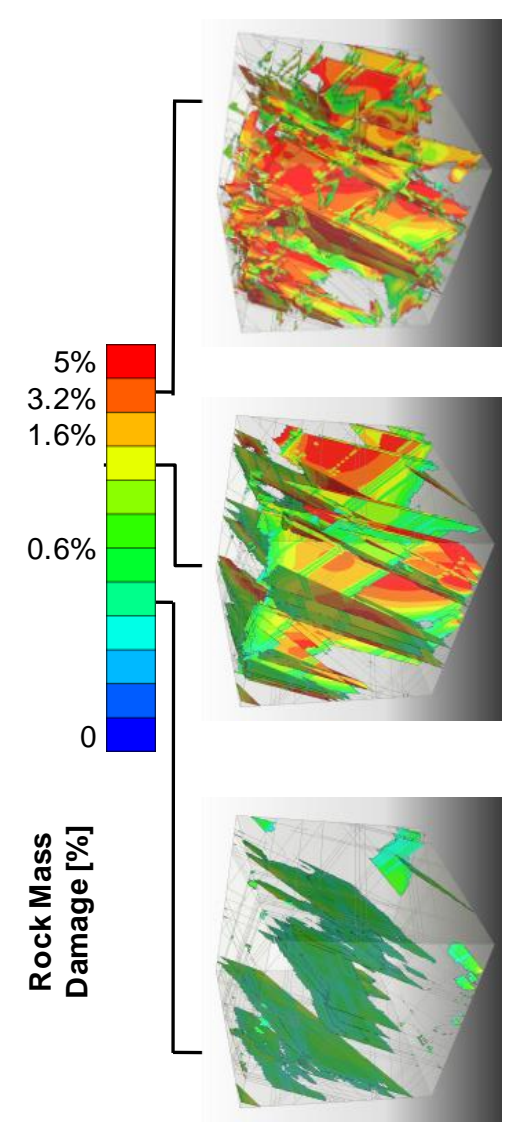

Figure 1 Damage scale used for LR2 hard rocks

3. Directly plotting a measure of rock mass damage as a model output differs from most traditional modelling workflows. Usually, damage is qualitatively inferred after the simulation by interpreting modelled stress or strength loss. This is done because the extent and magnitude of damage or deformation is expected to be underestimated by such models, and a direct comparison with visual observations of damage in the mine would be difficult. The difficulty with this approach is that it is also difficult to estimate the extent to which underestimating damage in any part of the mine influences deformation and stability of other parts, because rock mass behaviour throughout the mine is a strongly coupled system.

The main effort of the LR2 calibration is to match the modelled and measured extent of damage, so that higher order effects and mechanical coupling between different areas of the mine can be better captured and understood.

Making damage information available to the engineer for ground truthing is a critical part of the process - the intent is that by comparing the observed damage to modelled damage, the global deformation and damage in the model can be continuously improved and the limits of the model better understood.

4. Discrete structures that are explicitly represented in the model are modelled with cohesive finite elements. Cohesive elements may have any valid constitutive formulation in LR2. Their purpose is to permit very large dislocations and separations on discontinuities while providing the correct kinematics of contact between the adjacent structure surfaces.

In LR2, faults and shear zones are free to dislocate and dilate and the fault surfaces can dilate and degrade, and if needed, particles may flow though this is not needed for this problem.

The main benefit of the LR2 implementation for discontinuities is that the mechanics and kinematics of the contacts between solid continuous parts bound by cohesive elements is very 
well resolved and robustly solved; that is, the numerical solution is very stable and the representation of the stress-strain behaviour within continuum parts of the rock mass need not be compromised in order to incorporate discontinuum behaviour even when a large number of non-planar, complex structures is included.

An example of large dislocation and rock mass distortion in an LR2 model is shown in Figure 2. In the NRS model, the strains are of course smaller, but the same constitutive and kinematic mechanisms can evolve.
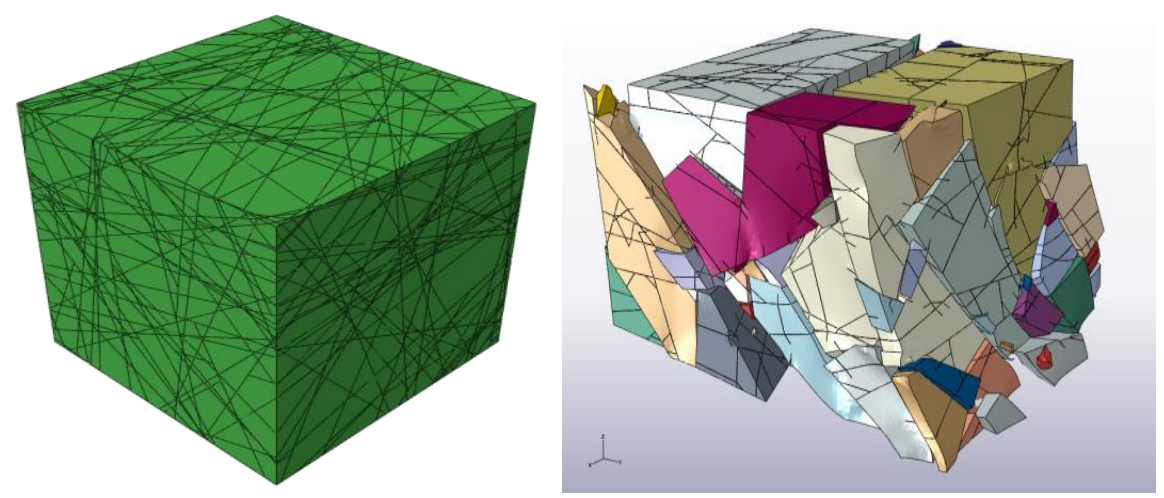

Figure 2 Example of large dislocation and rock mass distortion in an LR2 model

5. As an effort is made to accurately capture the extent and magnitude of rock mass damage, seismicity can be interpreted for both calibration and forecasting stages using energy and damage terms:

a. Nucleating seismicity:

- Occurs in slightly damaged rock or slightly yielded faults (Early Stage II damage, Figure 3 ) that are softening but not unstable so continue to store large amounts of energy, without becoming unstable at the scale of the Representative Elementary Volume (REV) scale - for the rock mass see (Stage II of Figure 3).

In the model, the simplest measure of nucleating seismic potential is high levels of stored energy in locations that are undergoing the first stages of yield (minor damage or Stage II). The model provides both state of yield energy release for this assessment.

- The model outputs energy in terms of specific energy release for a model step $\left(\mathrm{J} / \mathrm{m}^{3}\right.$ for rock mass release and $\mathrm{J} / \mathrm{m}^{2}$ for faults. The correlation between this value and seismicity is expressed with units events/volume or area/month or as an average inter-event distance.

- The nucleating measure correlates mostly with the low level microseismicity that is poorly clustered, e.g. 'background' noise.

b. Coalescing seismicity:

- Occurs as the rock mass and faults degrade and stress is redistributed, during later Stage II and Stage III damage as shown in Figure 3. During these phases the material undergoes more significant strength changes and eventually becomes unstable, so the degradation has a larger effect on the system as a whole.

- The units of the model output are $\mathrm{J} / \mathrm{m}^{3}$ for the rock mass and $\mathrm{J} / \mathrm{m}^{2}$ for faults. The correlation between these values and seismicity are expressed with units events/volume or area/month or as an expected average inter-event distance.

○ The measure correlates mostly with the rapidly growing or intensifying clusters of seismicity and larger event potential. 


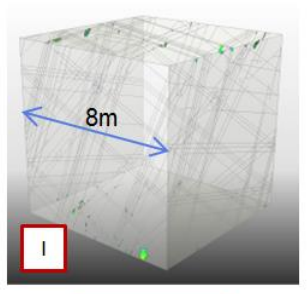

None

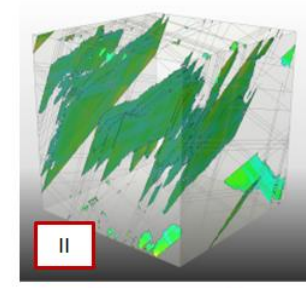

Minor

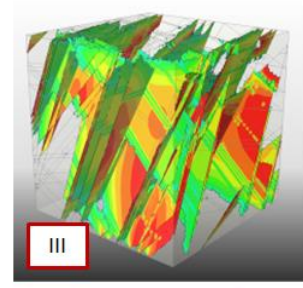

Moderate

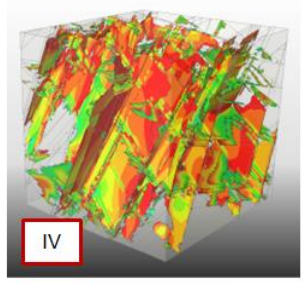

Sign.

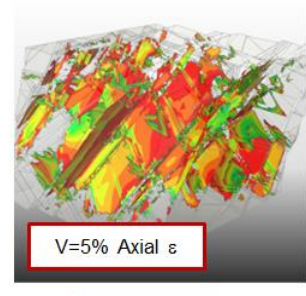

Very Sign.

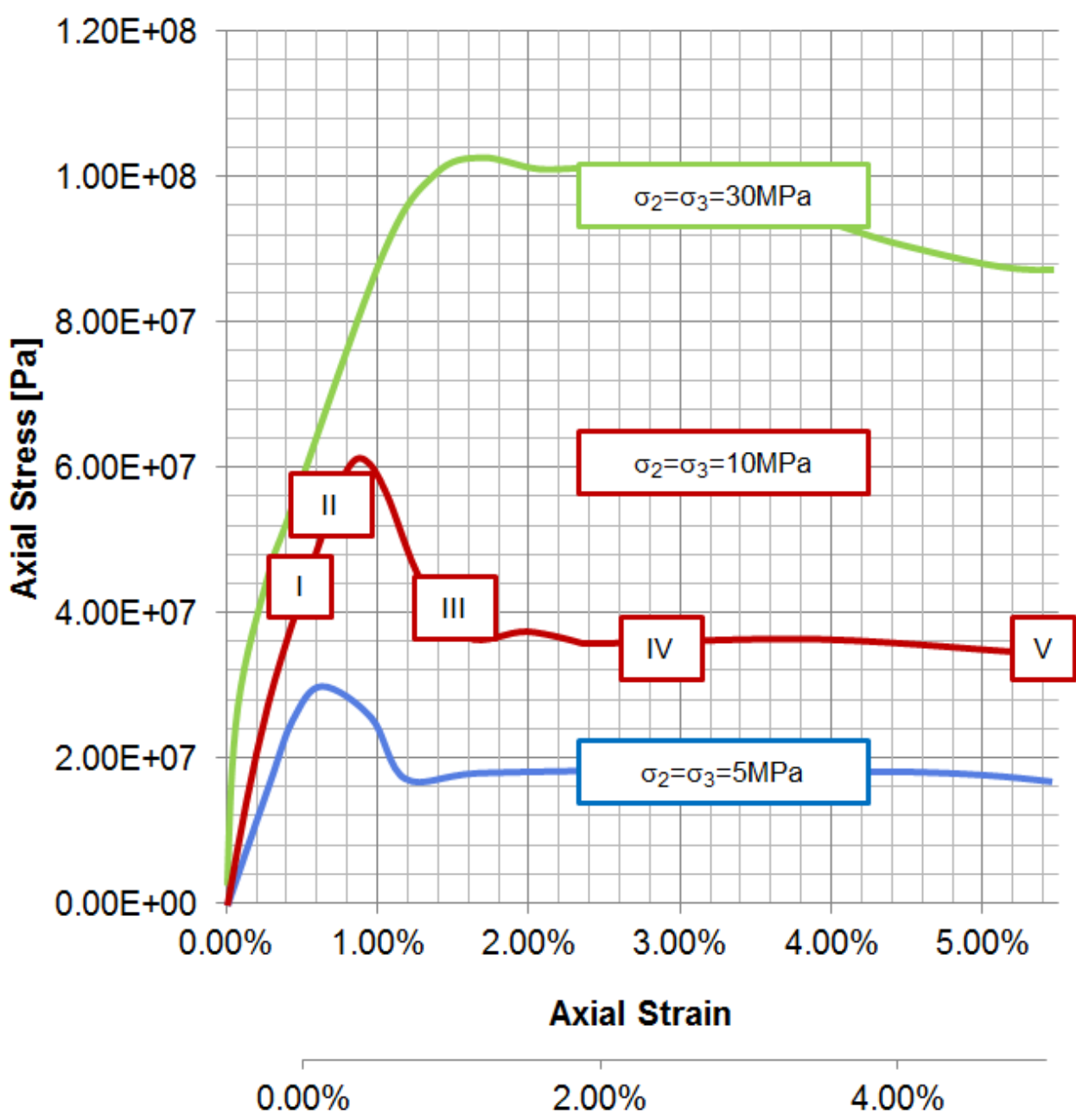

Equivalent Plastic Strain

Figure 3 Example stages of rock mass damage at a rock mass scale. These are the same stages used to interpret and describe rock mass damage in this report

The different phases of seismicity need not be decomposed into nucleating and coalescing parts, but doing so helps the mine to understand the state of the rock mass, and therefore make better decisions around management of the seismic hazard.

\section{2 Faults at NRS}

The faults are represented as explicit discontinuities in the NRS model - they define blocks that can slide and separate. The coupling of a model that can accommodate discontinuous deformation, as well as simulate the gradual degradation of the rock mass is very important for capturing the evolution of stress, seismicity and strain in the working areas. 
The complete 3D structural model was included as provided by the mine. A 3D perspective view of the model indicating the resolution of structure in the model is shown in Figure 4.

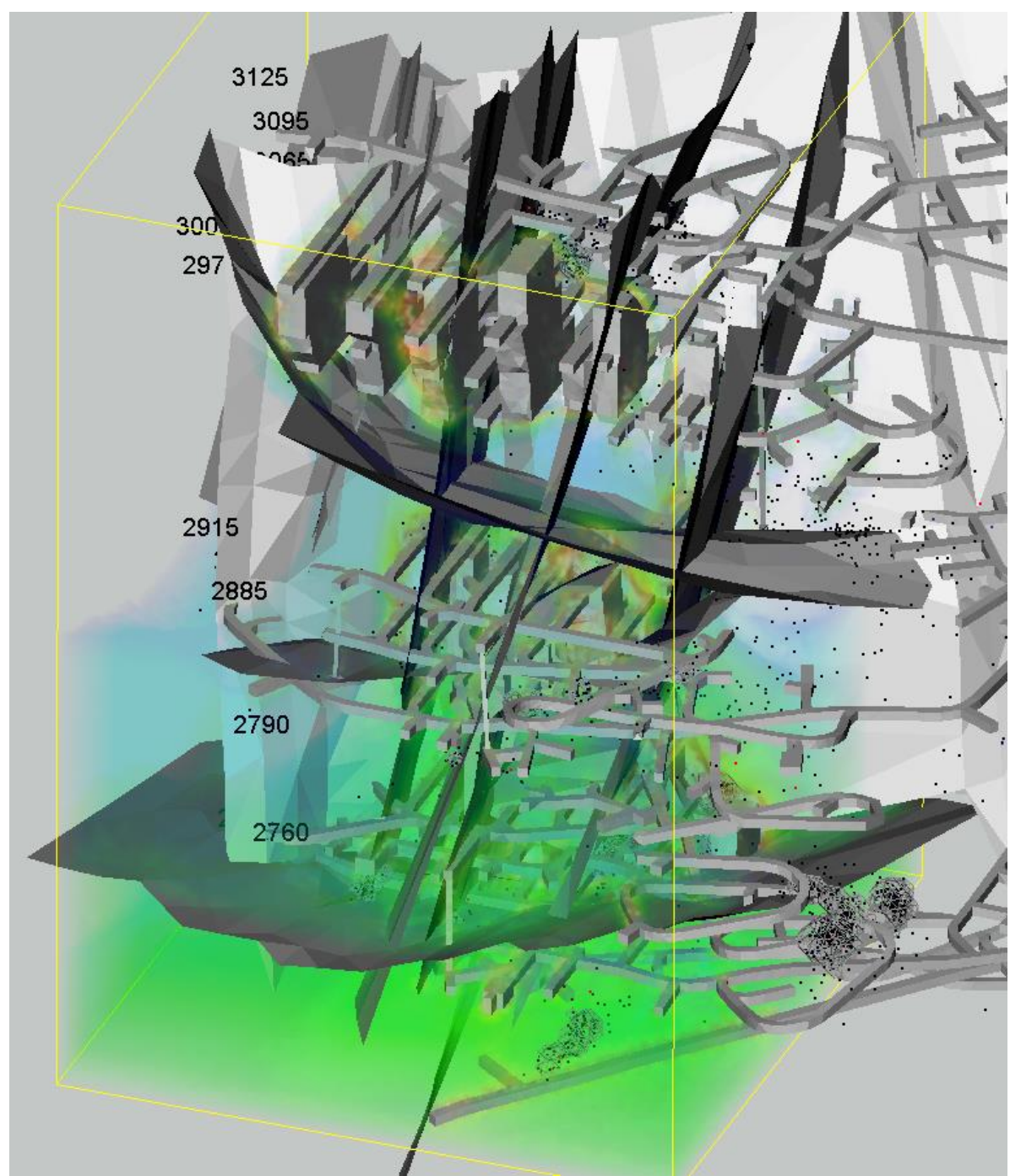

Figure 4 Image showing fault geometries as built in the model

\section{3 NRS geological domains}

The mine's interpreted geological domains were built into the model and treated as geotechnical domains for calibration purposes: each one has a unique set of material properties and a relationship with seismic potential. The domain boundaries were built explicitly using the geometry provide by the mine. 
Table 1 Summary of model features

\begin{tabular}{|c|c|c|}
\hline Feature & \multicolumn{2}{|l|}{ Summary } \\
\hline \multirow[t]{3}{*}{ Model type } & \multicolumn{2}{|c|}{ 3D, strain softening, dilatant, Explicit Finite Element } \\
\hline & \multicolumn{2}{|c|}{ Higher order tetrahedral elements for rock units } \\
\hline & \multicolumn{2}{|c|}{ Higher order cohesive elements for explicit structures } \\
\hline Simulation package & \multicolumn{2}{|l|}{ Abaqus 6.8 Explicit } \\
\hline \multirow[t]{4}{*}{$\begin{array}{l}\text { Constitutive model for } \\
\text { the rock mass }\end{array}$} & \multirow[t]{2}{*}{ Rock mass yield model: } & $\begin{array}{l}\text { Menetrey and William (1995) with eccentricity of } 0.6 \\
\text { to approximate the Hoek-Brown yield surface }\end{array}$ \\
\hline & & Applied for each geological unit \\
\hline & Plastic strain potential: & Menetrey and William (1995) \\
\hline & Softening: & $\begin{array}{l}\text { Piecewise as a function of strain for dilation, } \\
\text { cohesion, friction (Menetrey and William, 1995) }\end{array}$ \\
\hline \multirow[t]{2}{*}{ Discontinuities } & \multirow[t]{2}{*}{ Cohesive Elements: } & $\begin{array}{l}\text { Major contacts between lithologies modelled as } \\
\text { combined cohesive/contact elements }\end{array}$ \\
\hline & & Mohr-Coulomb yield criteria \\
\hline Sequencing & \multicolumn{2}{|c|}{ 200 extraction steps, as provided by the mine } \\
\hline Filling & \multicolumn{2}{|c|}{ Stopes left open for one step then tight filled } \\
\hline
\end{tabular}

\section{Calibration}

The calibration procedure to achieve a match between modelled and measured seismicity and comprises three stages:

- 1st calibration stage: Selection and bracketing.

The selection and bracketing stage aims to appreciate the problem, to identify the governing physics that must be captured, to select the modelling approach, to set model fundamentals and sufficiency requirements and then ultimately to identify initial model variables.

At the end of this stage, the model is run with base case, empirical material properties. Bold adjustments to material properties are made until the model brackets observed behaviour.

- 2nd calibration stage: Detailed fine adjustments.

The detailed fine adjustment stage aims to further refine the model fundamentals and variables, to improve precision and qualify or quantify the model error.

An example of a detailed fine adjustment is the incremental adjustment of the frictional strength of a particular fault in the model, to better match seismic measurements of movement on the fault.

At the end of this stage, the model variables are largely selected and the model is said to be calibrated.

- 3rd calibration stage: Interpretation and benchmarking.

The interpretation and benchmarking stage aims to aid model interpretation by identifying relationships between modelled quantities that are related to, but not direct measures of expected real world performance.

An example is the correlation between the modelled Rate of Energy Release (RER) and measured seismic event probability; RER is measured in $\mathrm{W} / \mathrm{m}^{3}$ or $\mathrm{W} / \mathrm{m}^{2}$ but event probability is measured in 
events $/ \mathrm{m}^{3} /$ month or events $/ \mathrm{m}^{2} /$ month. The expected nature of this correlation can be used to assist in model calibration, and a close correspondence can be achieved, but a final interpretation of the relationship is needed to convert the modelled value in to a real-world estimate of performance. When complete, the established benchmarks, or correlations for interpreting the calibrated model are established.

\section{$4 \quad$ Calibrated material properties}

Seven material property sets were used in the detailed fine adjustment stage. All seven model runs and input data are retained as a record of the sensitivity of the mine to changes in model inputs, and may be useful for considering deviations between modelled and forecast seismicity.

The main modelled-measured data incongruities in the rejected sets that drove the evolution towards the final property set were:

- Mismatches in seismicity across the geological domains - an insufficient transition between seismic behaviour across domains.

- Specific measured seismic clusters associated with the strength loss in pillars, or the attainment of a critical span of extracted stopes. These transitions appear as a the 'blowing out' of an event cluster that was previously associated with an advancing row of stopes, to a larger cluster spanning a larger group of stopes.

- The timing of seismicity on structures.

The final material property set is shown in Table 2. Note that $d$ is a dilation parameter for the LR2 model, not the Hoek-Brown disturbance factor.

Table 2 HB06 (final calibrated) rock mass scale material properties

\begin{tabular}{lcccc}
\hline & UCS $(\mathrm{Pa})$ & Eccentricity & $\mathbf{1 / a}$ & $\mathbf{N}$ \\
& $\mathbf{1}$ & $\mathbf{2}$ & $\mathbf{3}$ & $\mathbf{4}$ \\
\hline Felsic norite & $7.23 \mathrm{E}+07$ & 0.6 & 1.99 & 3 \\
Dark norite breccia & $5.99 \mathrm{E}+07$ & 0.6 & 1.99 & 3 \\
Late granite breccia & $9.35 \mathrm{E}+07$ & 0.6 & 2.00 & 3 \\
Felsic gneiss & $2.13 \mathrm{E}+08$ & 0.6 & 2.00 & 3 \\
Fault & $6.00 \mathrm{E}+07$ & 0.6 & 1.98 & 3 \\
Copper zones & $6.50 \mathrm{E}+07$ & 0.6 & 2.00 & 3 \\
\hline
\end{tabular}


Table 3 (continued) HBO6 (final calibrated) rock mass scale material properties

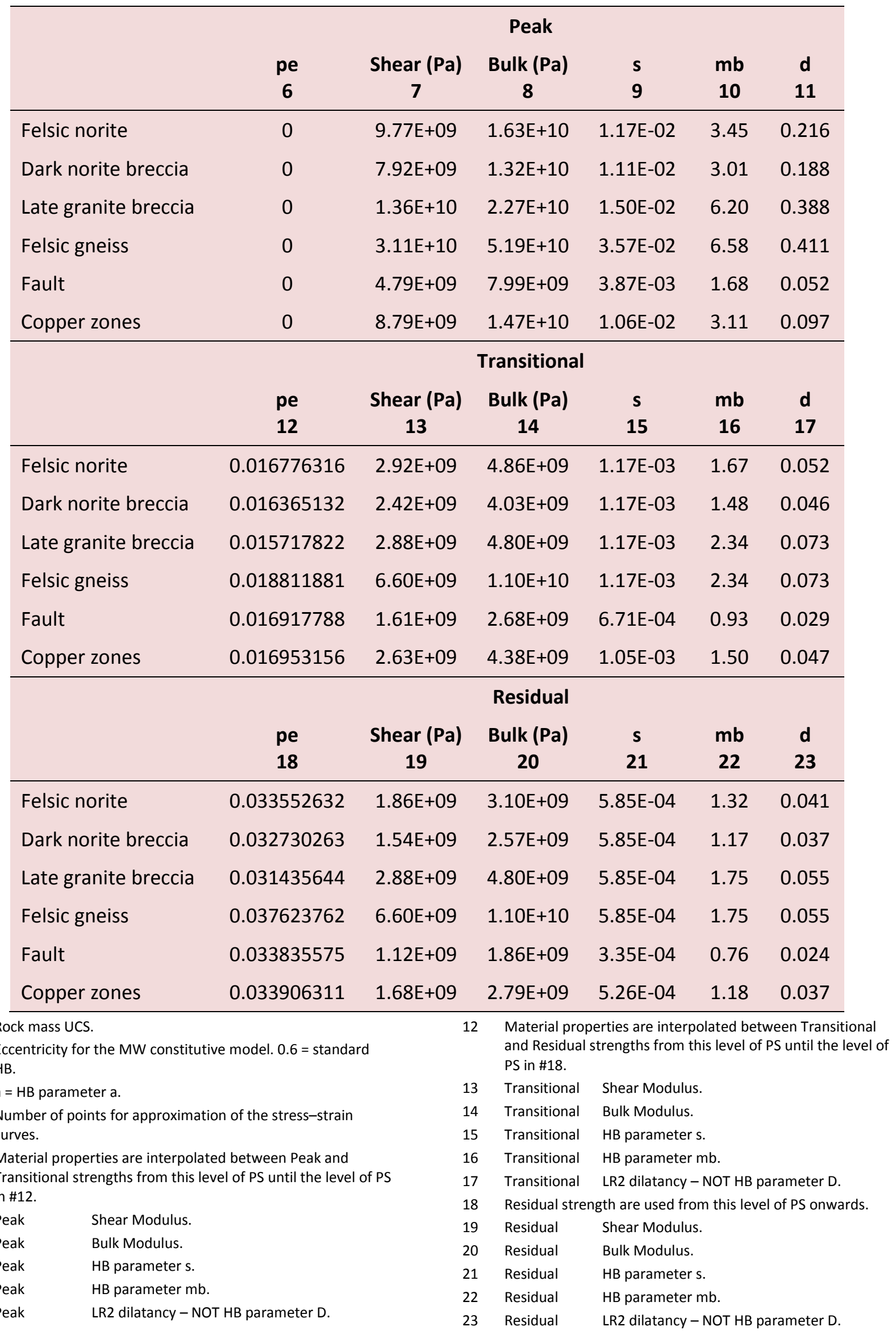




\section{1 Validation of material properties during calibration}

The validation of the final material property set is based on the comparison of modelled and measured seismicity for the calibration period. This is done by comparing the intensity of events within clusters of seismicity to the modelled measures of nucleating, coalescing and fault slip seismicity, as shown in Figures 5, 6 and 7. The figures show measured event clusters as wireframes, and various measures of modelled energy release as volume rendered clouds. To easily identify the mechanism generating each cluster in the image, the peak release for fault slip in was coloured purple, while the peak release for rock mass or nucleating seismicity was coloured red.
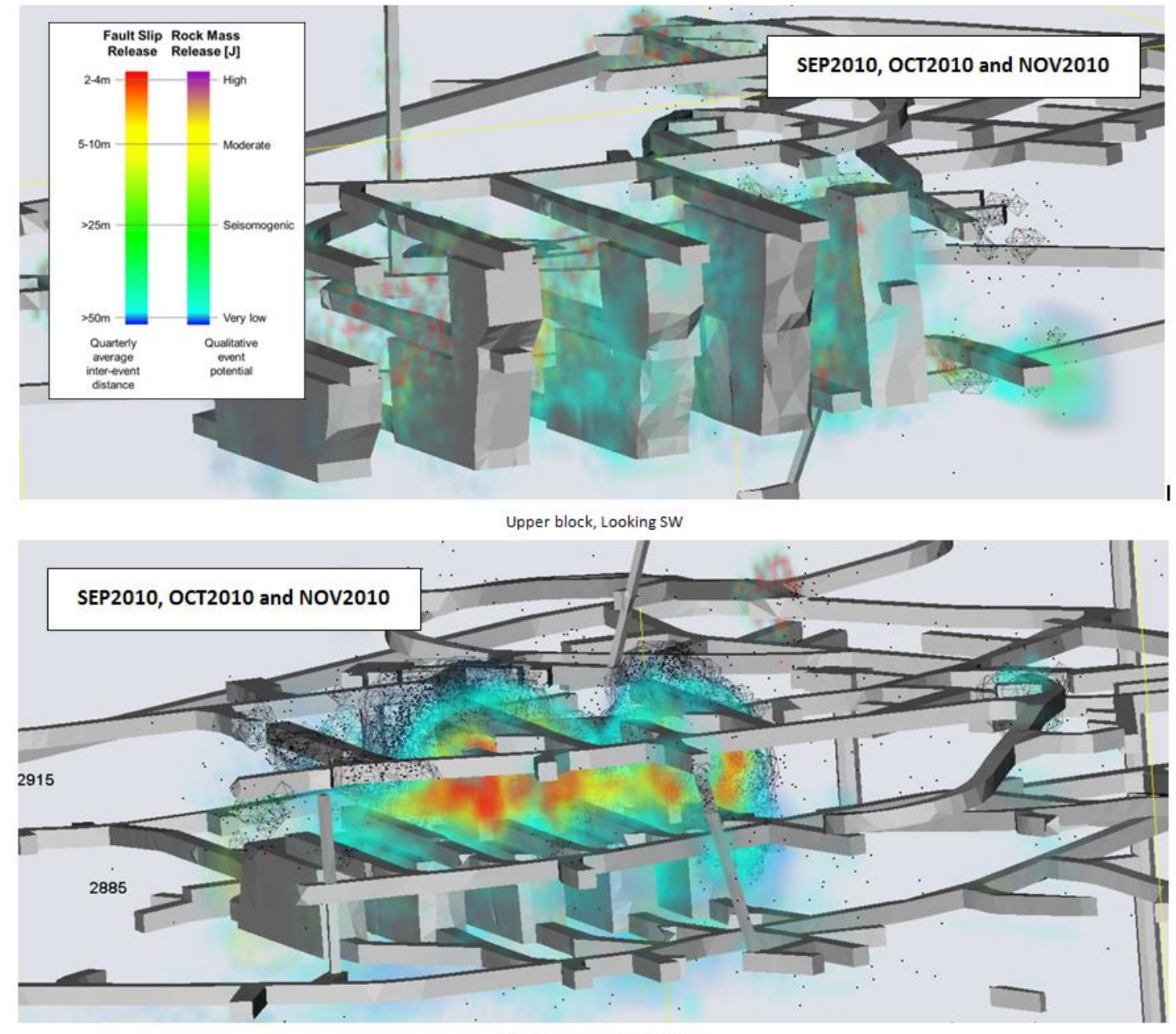

Middle Block, Looking SW

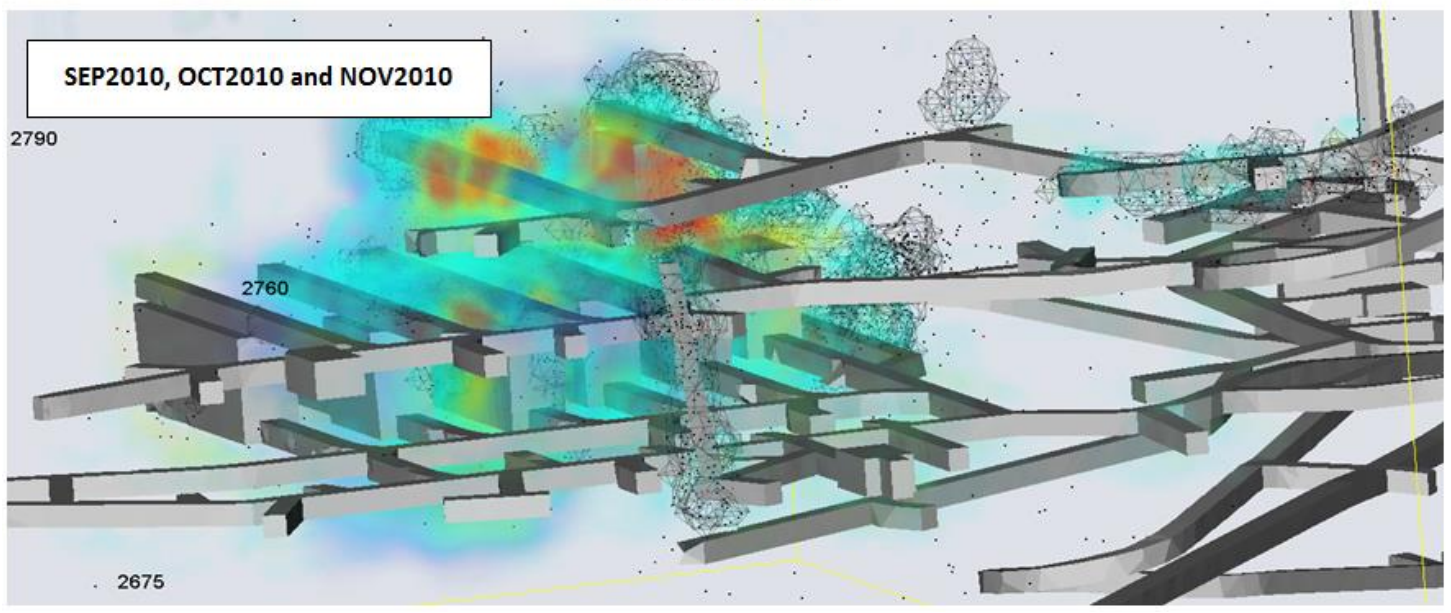

Figure 5 Modelled energy release versus seismicity, 30AUG2010 to 29 N0V2010 
The event clusters are inter-event (IE) distance isosurfaces of approximately 5-10 m. For this study, high intensity seismic activity was defined as a cluster with an IE distance of $<5 \mathrm{~m}$. Moderate intensity corresponded to an IE distance of $<10 \mathrm{~m}$, seismogenic zones i.e. continuous and bounded regions of increased activity above random, or background microseismicity was assessed by volumes with an IE distance of $<25 \mathrm{~m}$. Areas with an IE distance of $>25 \mathrm{~m}$ were considered to experience low, random or background noise only, possibly associated with poorly located small magnitude events, or larger events beyond the sensor array.

The modelled RER, shown as volume rendered clouds is either fault slip release, rock mass energy release or nucleating damage as defined in Section 2.

To establish a quantitative relation between RER and seismic potential, various isosurfaces were compared to various RER levels until the estimated best fit shown in the images was found. This relationship is approximate, but is a guide to expected seismic intensity and is shown in the legend of each figure. An alternative approach to the correlation of RER and event probability is the Cell Evaluation Method (Beck and Brady, 2002). In that case a statistical estimate of the fit of modelled RER to measured event probability would be obtained.

For the present study, the detailed comparison spans March 2010 to May 2011, although the model starts before any stoping or development takes place. A comparison of the modelled and measured data by quarter is summarised qualitatively in Table 4, where sufficiency is based on the assessment of the mine rock mechanics engineer.

Table 4 Qualitative classification of the fit between modelled energy release and event occurrence, based on the models intended use for assessing global deformation problems (UE = underestimate)

\begin{tabular}{|c|c|c|c|c|c|c|}
\hline \multirow[t]{2}{*}{ Period } & \multicolumn{2}{|c|}{ Upper Block } & \multicolumn{2}{|c|}{ Middle Block } & \multicolumn{2}{|c|}{ Lower Block } \\
\hline & Cluster & Background & Cluster & Background & Cluster & Background \\
\hline $\begin{array}{l}\text { MAR 2010- } \\
\text { MAY } 2010\end{array}$ & Sufficient & Sufficient & Sufficient & Sufficient & UE & Sufficient \\
\hline $\begin{array}{l}\text { JUN 2010- } \\
\text { AUG } 2010\end{array}$ & Sufficient & Sufficient & Sufficient & Sufficient & UE & Sufficient \\
\hline $\begin{array}{l}\text { SEP 2010- } \\
\text { NOV } 2010\end{array}$ & Sufficient & Sufficient & Sufficient & Sufficient & Sufficient & Sufficient \\
\hline $\begin{array}{c}\text { DEC 2010- } \\
\text { FEB } 2011\end{array}$ & Sufficient & Sufficient & Sufficient & Sufficient & Sufficient & Sufficient \\
\hline $\begin{array}{l}\text { MAR 2011- } \\
\text { MAY } 2011\end{array}$ & Sufficient & Sufficient & $\begin{array}{l}\text { Sufficient except } \\
\text { in Ramp - See } \\
\text { circle in Figure } 7\end{array}$ & Sufficient & Sufficient & Sufficient \\
\hline
\end{tabular}

Cross-checking modelled energy release and seismicity in each assessed period indicated a close match between the dense event clusters and the intense modelled energy release correlated with high event probability for most mining areas across most model steps. Early in the assessment period, the timing of certain high intensity clusters was offset from the actual timing for the lowest block, and this appears to be due to the lack of some structures in the fault model for that block. The issue highlights that the model is best used for global simulations, and the end user needs to account for the possibility that certain structures have not been mapped, but which may pose a significantly elevated seismic risk. 


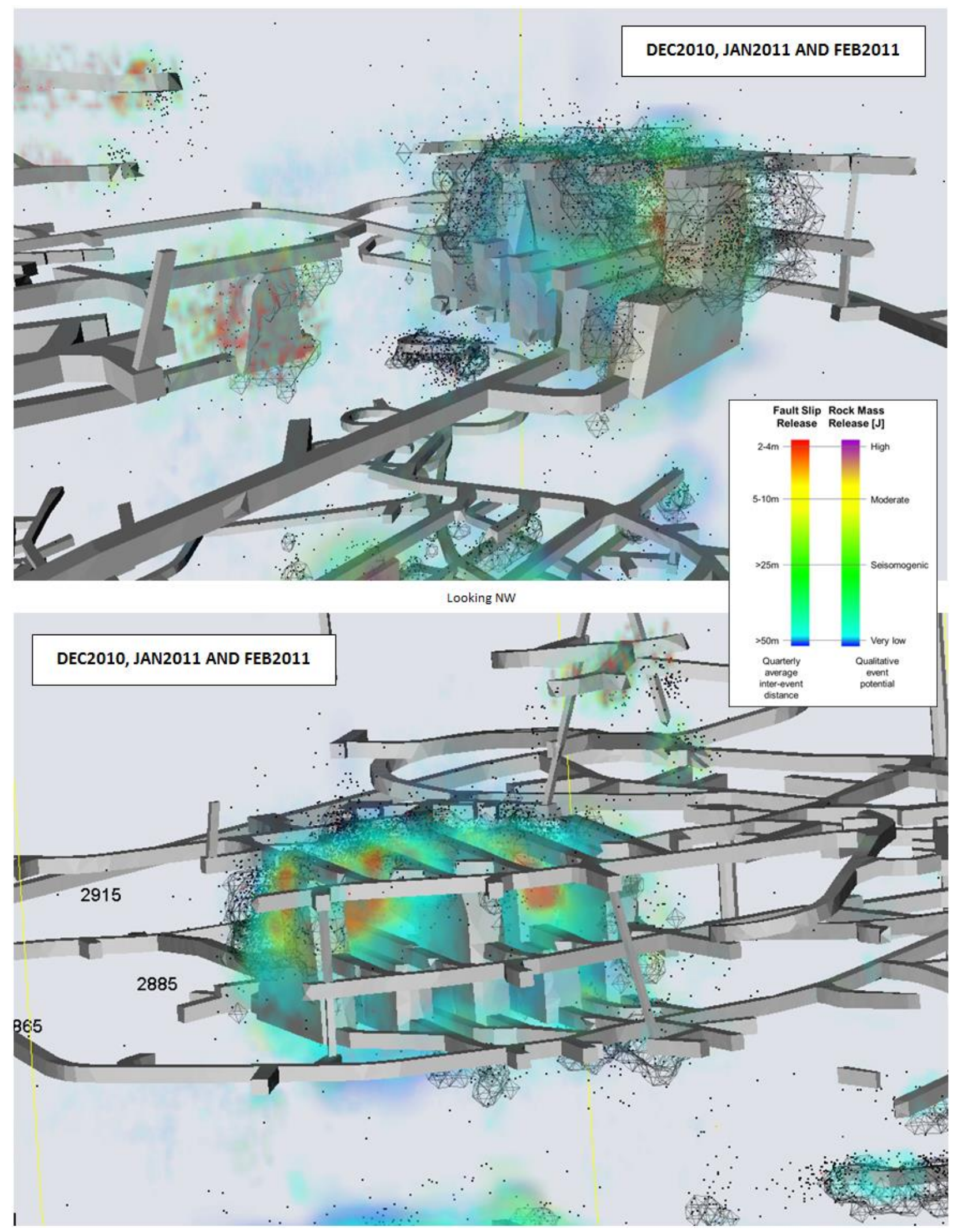

Figure 6 Modelled energy release versus seismicity, 30NOV2010 to 28FEB2011, Middle Block 

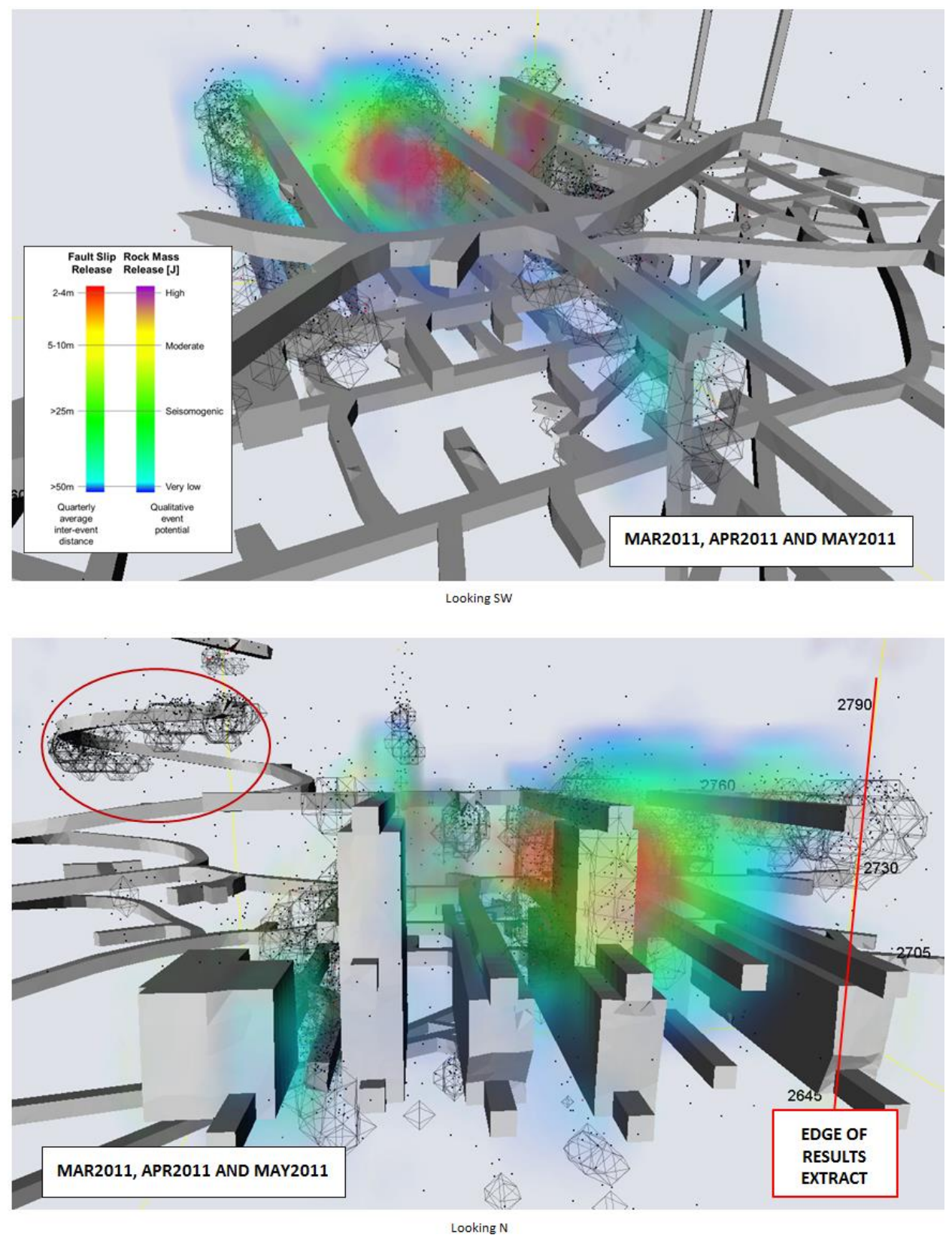

Figure 7 Modelled energy release versus seismicity, 28FEB2011 to 30MAY2011, Lower Block

After this comparison, it was concluded that the model was fit for the purposes of simulating global seismic phenomena at NRS, with potentially higher resolution in some areas.

An experience based assessment is that the level of calibration benchmarks well against other mines, and that the model could first be improved by incorporating smaller length scale structures and by simulating smaller extraction steps, possibly by breaking stopes into individual firings. This has been undertaken at some mines for a modest improvement in model resolution.

A model with this resolution also highlights areas where additional data collection is required to provide input to subsequent modelling phases to improve resolution and forecast reliability. Developing a high resolution calibrated model is an ongoing process, requiring an ongoing engineering programme comprising mapping, rock mass characterisation, testing, measurement, back analysis of significant events and re-calibration using several models during the life of a mine. Model results are used to identify further data collection requirements to be used in the next modelling phase, and so on. 


\section{$5 \quad$ Forward prediction and ground truthing example}

After calibration was complete, the model was used for forecasting. This allows comparison of the modelled and measured data for a true predictive period, and the result is shown in Figure 8 . This shows an equivalent quality of match between modelled and measured seismicity to the calibration period and importantly indicates that the model captures evolving phenomena not present in the calibration steps, including some seismogenic bridging through lower sills and substantive fault slip energy releases on previously low activity structures. Changes to the development schedule cause some mismatch between the model and measured seismicity for clusters due solely to new development.

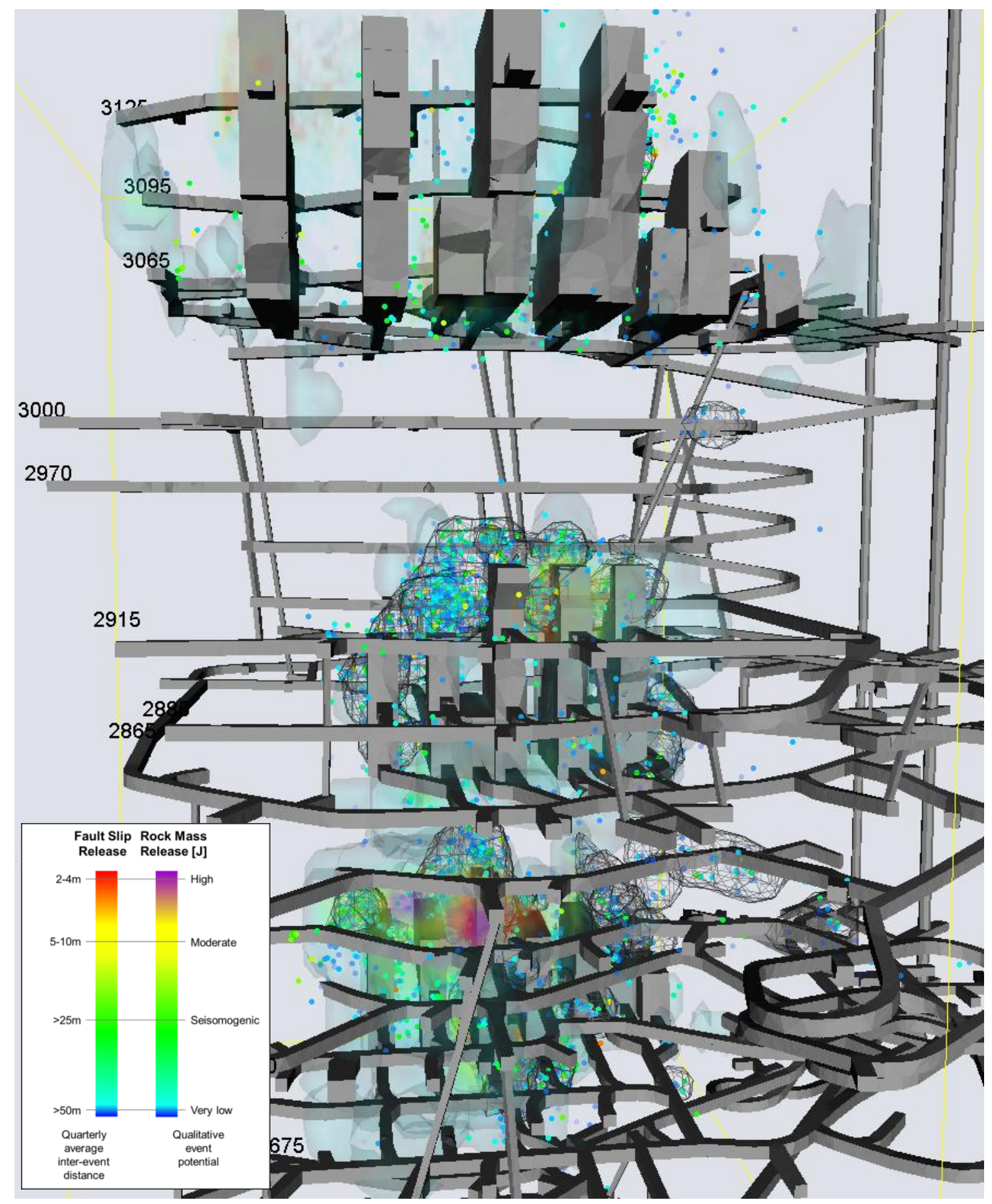

Figure 8 Modelled forward predicted energy release compared to measured seismicity. Measured events shown as coloured dots by magnitude and wireframe meshes of statistically identified clusters 


\section{Conclusions}

The discontinuum LR2 model of NRS is well calibrated for global scale assessments of induced deformation and seismic potential. The reasons why the model can match the measured seismicity sufficiently, are the careful matching of the actual and modelled extraction sequencing, the large strain, strain softening constitutive formulation, the incorporation of such a large number of explicit structures and practically, a high standard for calibration.

The tool does not replace any other component of sound mine design, day-to-day geotechnical practice or seismic risk management; rather it is a rigorously and continuously tested adjunct to the existing workflow to optimise the mine design and sequence to minimise safety and production risks.

To apply models like this, mines should consider the uncertainties of the model and consider the meaning of these uncertainties for the model outcomes when interpreting the results, but the potential to integrate these type of results to aid better decision making is an important opportunity.

Models of this type are improved by:

- Adding underground damage mapping and other comprehensive deformation data, such as passive tomography surveys to the calibration process. Point measurements such as extensometers also have value, but being local to a small excavation cannot substantially influence global input parameters unless undertaken on a wide scale throughout the mine.

- Adding smaller scale structures.

- Sub-dividing the geotechnical domains further with mapping data.

- Sequencing mining with smaller steps, possibly down to the scale of individual stope blasts.

- Making additional stress measurements, then calibrating the model to match the variations in stress across the mine.

- Ensuring that the seismic system provides adequate coverage of the mine, which could involve installing extra sensors to fill gaps if they exist.

\section{References}

Beck, D.A. and Brady, B.H.G. (2002) Evaluation and application of controlling parameters for seismic events in hard-rock mines, International Journal of Rock Mechanics and Mining Sciences, editorial reference: 2002/002093.

Levkovitch, V., Reusch, F. and Beck, D. (2010) Application of a non-linear confinement sensitive constitutive model to mine scale simulations subject to varying levels of confining stress, Rock Mechanics in Civil and Environmental Engineering, J. Zhao, V. Labiouse, J.P. Dudt, J.F. Mathier (eds), CRC Press, pp. 161-164.

Menetrey, P. and William, K. (1995) Triaxial failure criterion for geomaterials and its generalization, ACl Structural Journal, Vol. 92(3), pp. 311-317. 
